\title{
Dementia and Dental Health Care
}

\author{
Dipayan D* \\ BDS, Department of Public Health Dentistry, Tamil Nadu Government Dental College \\ and Hospital, India
}

*Corresponding author: Dipayan D, BDS, Department of Public Health Dentistry,

Tamil Nadu Government Dental College and Hospital, Tamil Nadu, India, 600 003, Tel:

\section{Editorial}

Volume 3 Issue 1

Received Date: January 20, 2018

Published Date: January 29, 2018

DOI: $10.23880 /$ oajds- 16000160

7358565184; Email: datta.dipayan2@gmail.com

\section{Editorial}

What is the first thing you remember to do while freshening up yourself after waking up in the morning? The commonest answer is tooth brushing. Until we clean our oral cavity, we don't feel the overall freshness at all. But there are people who don't brush their teeth daily not because they don't want to do, but because they forget to do.

Dementia is said to be a neurodegenerative disease that results in deterioration in memory as well as reasoning and communication skills. In case the disease progresses, different psychological symptoms like depression, aggression, psychosis etc. can also be found in the patients' behaviour. Alzheimer's disease and Parkinson's disease are two of the neurological disorders that are responsible for dementia.

Taking good care of dental health of persons with dementia is of utmost importance. The reasons are:

a) They frequently forget the procedure of complex motor tasks like tooth brushing, inserting dentures etc. Sometimes they cannot remember where they have kept their dentures.

b) Many patients with dementia suffer from other comorbid psychological conditions too. To reduce their hallucinations, delusions, aggressiveness etc. they are often given antipsychotic medications which has various adverse effects on oral tissues like xerostomia, altered taste sensation etc.

c) As their cognitive abilities are declined, it often becomes difficult to make them understand the importance of oral hygiene practice.

Oral cavity which has been left uncleaned for a considerable period of time will invariably be affected by different dental diseases. Persons suffering from dementia who do not maintain their oral hygiene have been found to have dental caries, periodontal diseases, tooth loss and difficulties with dentures due to dryness of mouth and improper swallowing pattern.

At present, no definitive treatment for dementia exists, hence, it inevitably affects the person's ability to perform day-to-day activities like regular oral hygiene practice leading to poorer oral health which even becomes worse in the absence of the care givers. So it is the responsibility of the family members as well as the Dental health care provider to take proper care of these people. Regular follow up is a must and all the necessary instructions should be given in front of the care takers so that later they can also help them to remember what they have been told. The Dental Surgeon should possess utmost patience and cautions during any Dental procedure in these patients as their mind might be prone to be unstable under any discomfort or stressful condition. Additionally, those patients who cannot help themselves without depending on others for care are at highest risk of being neglected. As a health care professional, the Dentist must be able to detect the alarming signs of elder abuse and act accordingly for their well-being.

Being a psychological condition, dementia does not only affect the mental and general health of the people, it also deteriorates their social position. The Dental health professional has the scope for improving their oral health as well their quality of life. Early diagnosis can aid in appropriate intervention through multidisciplinary approaches to achieve long-term positive oral health outcomes. 